\title{
Orion revisited
}

\section{The massive cluster in front of the Orion nebula cluster ${ }^{\star}$}

\author{
J. Alves ${ }^{1}$ and H. Bouy ${ }^{2}$ \\ 1 Department of Astrophysics, University of Vienna, Türkenschanzstrasse 17, 1180 Vienna, Austria \\ e-mail: joao.alves@univie.ac.at \\ 2 Centro de Astrobiología, INTA-CSIC, PO Box 78, 28691 Villanueva de la Cañada, Madrid, Spain \\ e-mail: hbouy@cab.inta-csic.es
}

Received 27 July 2012 / Accepted 21 September 2012

\begin{abstract}
Aims. The aim of this work is to characterize the stellar population between Earth and the Orion A molecular cloud where the wellknown star formation benchmark Orion nebula cluster (ONC) is embedded.

Methods. We used the denser regions the Orion A cloud to block optical background light, effectively isolating the stellar population in front of it. We then used a multi-wavelength observational approach to characterize the cloud's foreground stellar population.

Results. We find that there is a rich stellar population in front of the Orion A cloud, from B-stars to M-stars, with a distinct 1) spatial distribution; 2) luminosity function; and 3) velocity dispersion from the reddened population inside the Orion A cloud. The spatial distribution of this population peaks strongly around NGC 1980 (iota Ori) and is, in all likelihood, the extended stellar content of this poorly studied cluster. We infer an age of 4-5 Myr for NGC 1980 and estimate a cluster population of about 2000 stars, which makes it one of the most massive clusters in the entire Orion complex. This newly found population overlaps significantly with what is currently assumed to be the ONC and the L1641N populations, and can make up for more than 10-20\% of the ONC population (30-60\% if the Trapezium cluster is excluded from consideration). What is currently taken in the literature as the ONC is then a mix of several intrinsically different populations, namely 1) the youngest population, including the Trapezium cluster and ongoing star formation in the dense gas inside the nebula; 2) the foreground population, dominated by the NGC 1980 cluster; and 3) the poorly constrained population of foreground and background Galactic field stars.

Conclusions. Our results support a scenario where the ONC and L1641N are not directly associated with NGC 1980, i.e., they are not the same population emerging from its parental cloud, but are instead distinct overlapping populations. The nearest massive star formation region and the template for massive star- and cluster formation models is then substantially contaminated by the foreground stellar population of the massive NGC 1980 cluster, formed about 4-5 Myr ago in a different, but perhaps related, event in the larger Orion star formation complex. This result calls for a revision of most of the observables in the benchmark ONC region (e.g., ages, age spread, cluster size, mass function, disk frequency, etc.).
\end{abstract}

Key words. stars: formation - HII regions - stars: pre-main sequence - stars: early-type - stars: late-type - dust, extinction

\section{Introduction}

The Orion nebula is one of the most often studied objects in the sky, with observational records dating back to about 400 years, coinciding with the early developments of the telescope (Muench et al. 2008). It is an object of critical importance for astrophysics as it contains the nearest $(400 \mathrm{pc})$ massive star formation region to Earth, the Orion nebula cluster (ONC; e.g. Johnson 1965; Walker 1972), which is the benchmark region for massive star- and cluster formation studies. Recent distance estimates to the Orion nebula using parallax put this object at about 400 pc from Earth $\left(389_{-21}^{+24}\right.$ pc, Sandstrom et al. 2007; $414 \pm 7$ pc, Menten et al. 2007; $437 \pm 19$ pc, Hirota et al. 2007; and $419 \pm 6 \mathrm{pc}$, Kim et al. 2008). Some of the most basic observables of the star formation process, such as 1) star formation rates (Lada \& Lada 1995; Lada et al. 2010); 2) star formation history (Hillenbrand 1997); 3) age spreads (Jeffries et al. 2011; Reggiani et al. 2011); 4) the initial mass function to the substellar

* Based on observations collected at the German-Spanish Astronomical Center, Calar Alto, jointly operated by the MaxPlanck-Institut für Astronomie Heidelberg and the Instituto de Astrofísica de Andalucía (CSIC). regime (Hillenbrand \& Carpenter 2000; Muench et al. 2002; Da Rio et al. 2012; Hsu et al. 2012); 5) the fraction, size distribution, and lifetime of circumstellar disks (Hillenbrand et al. 1998; Lada et al. 2000; Muench et al. 2001; Vicente \& Alves 2005); 6) their interplay with massive stars (O'dell et al. 1993), binarity (Petr et al. 1998; Köhler et al. 2006), rotation (Herbst et al. 2002), magnetic fields (Feigelson et al. 2003); and 7) young cluster dynamics (Hillenbrand \& Hartmann 1998; Fúrész et al. 2008; Tobin et al. 2009), have all been derived from this benchmark region (see the meticulous reviews of Bally 2008; Muench et al. 2008; O'Dell et al. 2008). Naturally, the ONC is also the benchmark region for theoretical and numerical models of massive and clustered star formation (Palla \& Stahler 1999; Klessen $\&$ Burkert 2000; Clarke et al. 2000; Bonnell et al. 2001; Bate et al. 2003; Tan et al. 2006; Huff \& Stahler 2006; Krumholz et al. 2011; Allison \& Goodwin 2011).

It is quite remarkable that within only $1.5^{\circ}$ of the ONC there are several contiguous, and likely overlapping, groups of young stars (see Fig. 1), although few studies have tackled the entire region as a whole. Unfortunately, the three-dimensional arrangement of star formation regions, in particular massive ones, is far from simple and is essentially unknown given the current 


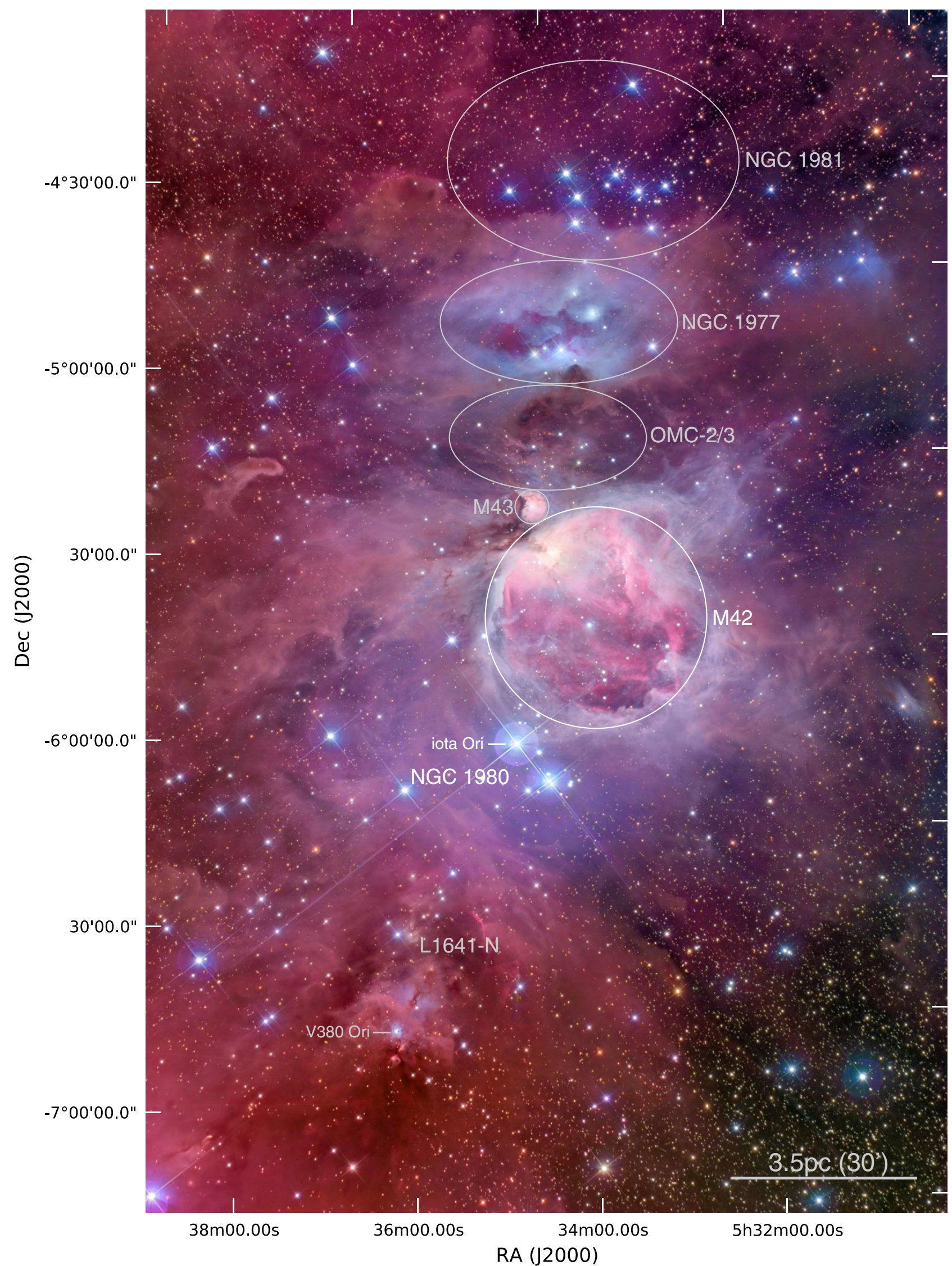

Fig. 1. Optical image of the north end of the Orion A molecular cloud, including the somewhat more evolved populations of NGC 1981, NGC 1977, and NGC 1980 (Orion OB 1c subgroup) and the Orion nebula cluster (Orion OB 1d subgroup), projected against the Orion nebula (M42). This image illustrates well the complicated distribution of young stars in the vicinity of the ONC, with scattered groups of more evolved blue massive stars projected against partially embedded groups of younger stars (M43, ONC, OMC-2/3, L1641N). Image courtesy of Jon Christensen (christensenastroimages.com). 
distance accuracy to even the nearest star formation regions. It is clear, however, that the ONC is partially embedded in its parental Orion A molecular cloud, which in turn is inside the large $\sim 200 \mathrm{pc}$ Orion star formation complex, where groups of young stars with ages from a few to about $10 \mathrm{Myr}$ are seen (Brown et al. 1994; Briceño et al. 2007). It has long been suspected that a more evolved group (subgroup Ori OB 1c, including NGC 1981 and NGC 1980, Blaauw 1964; Warren \& Hesser 1978 ) is in the foreground of the molecular cloud in which the younger ONC population (subgroup Ori OB 1d) is still partially embedded (see Gomez \& Lada 1998, for a large-scale analysis of the possible interplay between these two subgroups).

There are two different views on the stellar population inside the Orion nebula. The first suggests that the core of the ONC, the Trapezium cluster, is an entity distinct from the rest of the stellar population in the nebula, while the second, more prevalent view, suggests that the Trapezium is instead the core region of a larger cluster emerging from the Orion nebula. Herbig \& Terndrup (1986) performed one of the first charge-coupled devices (CCD) observations of an area centered on the Trapezium cluster (covering $\sim 9.2^{\prime 2}$ ), and from the exceptional high stellar density found they argued that the Trapezium cluster was an entity distinct from the surrounding stellar population, including the stellar population inside the Orion nebula. An opposite view was proposed by Hillenbrand \& Hartmann (1998), who compared optical and near-infrared surveys of the ONC with virial equilibrium cluster models to argue that the entire ONC is likely a single young stellar population.

Confirming which view is correct is critical because they imply different formation scenarios for the ONC, and assuming the ONC is typical, different scenarios for the formation of stellar clusters in general. While the first view implies a bursty formation of the bulk of the stars in a relatively small volume of the cloud, the second, by assuming a more extended cluster, calls necessarily for a longer and more continuos process, allowing for measurable age spreads in the young population, and for substantial fractions of young stellar objects (YSOs) at all evolutionary phases, from Class 0 to Class III. Observationally, the first view argues that the Orion OB 1c subgroup is a star formation event distinct from the 1d subgroup while the second and more prevalent view argues that the two subgroups are the same population, i.e., the Ori OB 1c subgroup is simply the more evolved stellar population emerging from the cloud where group $1 \mathrm{~d}$ still resides.

If the first view prevails, i.e., if the Trapezium cluster and ongoing star formation in the dense gas in its surroundings represent a population distinct from the rest of the stars in the larger ONC region, then what is normally taken in the literature as the $\mathrm{ONC}$ is likely to be a superposition of different stellar populations. If this is the case, then the basic star formation observables currently accepted for this benchmark region (e.g., ages, age spread, cluster size, mass function, disk frequency, etc.) could be compromised.

In this paper we address this important question by attempting to characterize the stellar populations between Earth and the Orion nebula. Our approach consists of using the Orion A cloud to block optical background light, effectively isolating the stellar population in front of it. We then use a multi-wavelength observational approach to characterize the cloud's and nebula's foreground population. We find that there are two clearly defined, distinct, and unfortunately overlapping stellar populations: 1) a foreground, "zero" extinction population dominated by the poorly studied but massive NGC 1980 cluster; and 2) the reddened population associated with the Trapezium cluster and
L1641N star-forming regions, supporting the first view on the structure of the ONC as described above. This result calls for a revision of most of the star formation observables for this fiducial object.

This paper is structured as follows. In Sect. 2 we describe the observational data acquired for this project as well as the archival data used. In Sect. 3 we present the results of our approach, namely the identification of the two foreground populations and their characterization. We present a general discussion on the importance of the result found in Sect. 4 and summarize the main results of the paper in Sect. 5.

\section{Data}

To characterize the foreground population of the Orion A molecular cloud we will make use of existing surveys together with raw data from Canada-France-Hawaii Telescope (CFHT), Calar Alto Observatory (CAHA $1.23 \mathrm{~m}$ ), and the Spitzer satellite, which were processed and analyzed for the purpose of this investigation.

\subsection{Catalogs}

We retrieved the astrometry and photometry for all sources within a box of $5^{\circ} \times 15^{\circ}$ centered around $\mathrm{RA}=85.7$ and Dec $=$ $-4^{\circ}$ (J2000) in the Sloan Digital Sky Survey III, the Wide-field Infrared Survey Explorer (WISE Cutri \& et al. 2012), the Third XMM-Newton serendipitous source (Watson et al. 2009) and the 2MASS catalogs (Skrutskie et al. 2006). Table 1 gives an overview of the properties of these catalogs.

\subsection{CFHT/Megacam}

A mosaic of $2 \times 2$ pointings covering $2^{\circ} \times 2^{\circ}$ centered on the ONC was observed with CFHT/Megacam (Boulade et al. 2003) with the Sloan ugr filters on 2005 February 14 (P. I. Cuillandre). Figure 2 gives an overview of the area covered by these observations. The conditions were photometric, as described in the Skyprobe database (Cuillandre et al. 2004). Seeing was variable, oscillating between $1-2^{\prime \prime}$ as measured in the images. A total of five exposures of $150 \mathrm{~s}$ ( $u$-band), $40 \mathrm{~s}$ ( $g$-band), and $40 \mathrm{~s}$ ( $r$-band) each were obtained at each of the four positions. The observations were made in dither mode, with a jitter width of a few arcminutes at each position. This allows filling the CCD-to-CCD and position gaps and correcting for deviant pixels and cosmic ray events. The images were processed using the recommended Elixir reduction package (Magnier \& Cuillandre 2004). Aperture photometry was then extracted using SExtractor (Bertin \& Arnouts 1996) and the photometric zeropoints in the SDSS system were derived by cross-matching with the SDSS catalog. The CFHT/Megacam observations complement the SDSS data in one critical aspect: they provide data for regions around bright stars and nebulae, in particular the Orion nebula region that is missing in the SDSS data.

\subsection{Calar Alto/1.23 m CCD camera}

Selected pointings of the ONC (see Table 2 and Fig. 2) were observed on 2011 December 15 with the Calar Alto CCD camera mounted on the $1.23 \mathrm{~m}$ telescope (hereafter CAHA123). The CCD camera is a $2 \mathrm{k} \times 2 \mathrm{k}$ optical imager with a $17^{\prime}$ field-ofview. The Sloan filters available at Calar Alto vignet the field and reduce it to a circular $11^{\prime}$ diameter field-of-view. These 


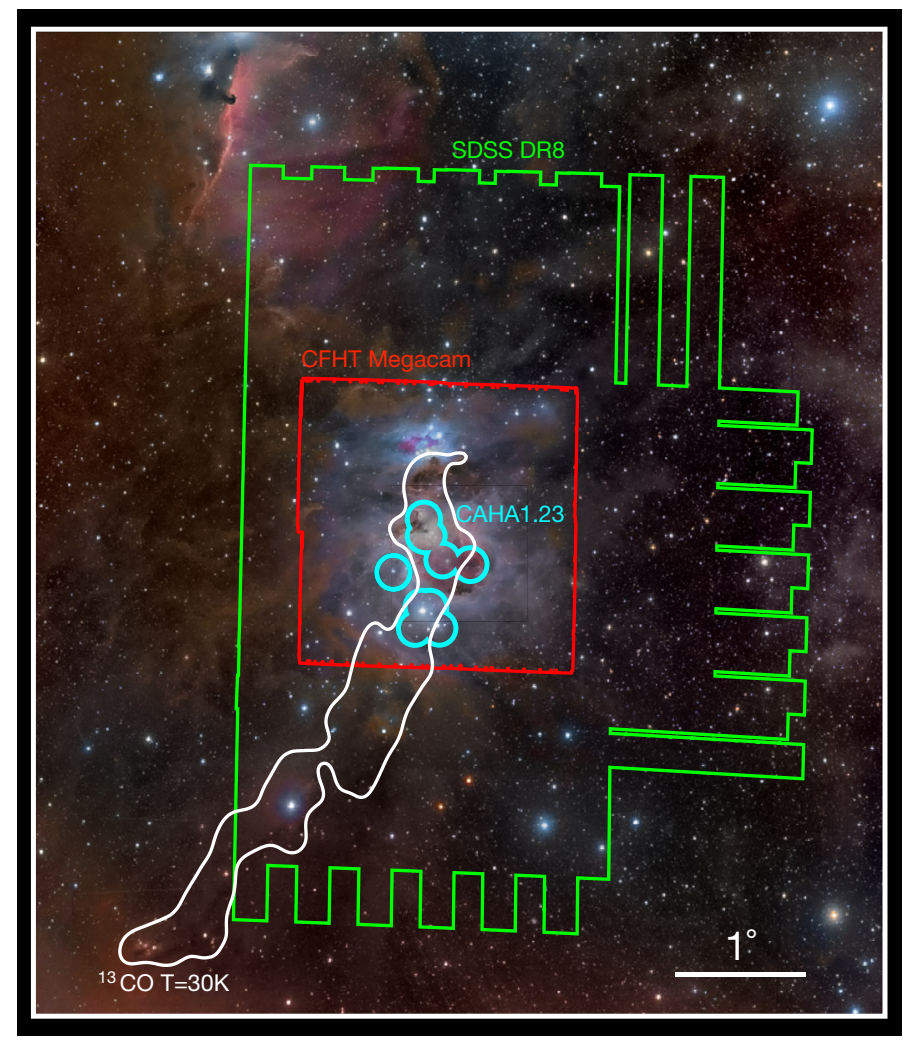

Fig. 2. Coverage of the optical datasets used in this study. The SDSS images used in this study are represented in green, the CFHT/Megacam images in red, and the Calar Alto $1.23 \mathrm{~m} \mathrm{CCD}$ observations are represented in light blue. The contour corresponding to an integrated intensity of ${ }^{13} \mathrm{CO}$ of $30 \mathrm{~K} \mathrm{~km} \mathrm{~s}^{-1}$ is represented in white. North is up and east is left. The angular scale is indicated in the lower left. Background photograph courtesy of Rogelio Bernal Andreo (DeepSkyColors . com).

Table 1. Catalogs and observations used in this study.

\begin{tabular}{lc}
\hline \hline Instrument & $\begin{array}{c}\text { Band/ } \\
\text { Channel }\end{array}$ \\
\hline XMM-Newton/EPIC & $0.1-10 \mathrm{keV}$ \\
SDSS & $u, g, r, i, z$ \\
CFHT/Megacam & $u, g, r$ \\
2MASS & $J, H, K \mathrm{~s}$ \\
WISE & $3.3,4.6,12,22 \mu \mathrm{m}$ \\
Spitzer/IRAC & $3.6,4.5,5.8,8.0 \mu \mathrm{m}$ \\
Spitzer/MIPS & $24 \mu \mathrm{m}$ \\
Calar Alto 1.23 m & $u, g, r$ \\
\hline
\end{tabular}

observations are meant to complement the CFHT and SDSS observations below their saturation limits (at $u g r \approx 12 \mathrm{mag}$ ), and in the vicinity of bright saturated stars. Short exposures of 0.1 and $5.0 \mathrm{~s}$ were obtained in the Sloan $g r$ filters, and of 0.1 and $10 \mathrm{~s}$ in the Sloan $u$ filter. The telescope was slightly defocused to avoid saturation of the brightest stars. Three standard fields (SA 97, SA 92 and BD+21D0607, Smith et al. 2002) were observed during the course of the night to derive accurate zeropoints. Each pointing was observed with a small dithering of a couple of arcminutes to correct for deviant pixels and cosmic ray events. The images were pre-processed (bias subtraction and flat-field correction) using standard procedure with the Eclipse reduction package (Devillard 1997). The astrometric registration and stacking were then performed using the AstrOmatic software suite (Bertin 2010a). Aperture photometry was finally
Table 2. CAHA 1.23 m CCD observations.

\begin{tabular}{lcc}
\hline \hline Field & RA $(\mathrm{J} 2000)$ & Dec $(\mathrm{J} 2000)$ \\
\hline Trapezium & $05: 35: 19.341$ & $-05: 23: 30.35$ \\
Field 1 & $05: 35: 24.651$ & $-05: 55: 06.69$ \\
Field 2 & $05: 34: 56.819$ & $-05: 59: 59.55$ \\
Field 3 & $05: 35: 25.044$ & $-05: 59: 15.44$ \\
Field 4 & $05: 34: 52.120$ & $-05: 34: 04.66$ \\
Field 5 & $05: 33: 59.980$ & $-05: 35: 40.17$ \\
Field 6 & $05: 36: 09.483$ & $-05: 38: 17.68$ \\
Field 7 & $05: 37: 23.116$ & $-05: 56: 10.97$ \\
\hline
\end{tabular}

extracted using SExtractor and the photometric zero-points in the SDSS system were derived by cross-matching with the SDSS and Megacam catalogs. The night was clear but not photometric. We observed a dispersion in the zero-point measurements through the night of $0.06 \mathrm{mag}$ in $u$ and $g$, and 0.16 mag in $r$, which we added quadratically to the photometric measurement uncertainties.

\subsection{Spitzer/IRAC}

The ONC has been extensively observed with IRAC on-board the Spitzer observatory in the course of programs 30641, 43, and 50070 . We retrieved the corresponding IRAC BCD images and associated ancillary products from the public archive and processed them following standard procedures with the recommended MOPEX software (Makovoz \& Marleau 2005). The observations were all made in the high dynamics range mode, providing short (0.6 s) and long (12 s) exposure. We processed the two sets independently so as to cover the widest dynamic range. The procedure within MOPEX includes overlap correction, resampling, interpolation (to have an output pixel scale of 0.6 ) and outlier rejection. The individual frames were then mediancombined with Swarp (Bertin 2010b) using the rms maps produced by MOPEX as weight maps. Aperture photometry of all the sources brighter than the 3- $\sigma$ noise of the local background was extracted using SExtractor. We verified that the corresponding photometry agrees well with the IRAC photometry from Fang et al. (2009) within the uncertainties.

\subsection{Spitzer MIPS1}

The ONC was observed with the Spitzer Space Telescope and its MIPS instruments in the course of programs 202, 30641 , $30765,3315,40503,47,50070$, and 58. We retrieved all corresponding MIPS1 $(24 \mu \mathrm{m}) \mathrm{BCD}$ images from the public archive and processed them with the recommended MOPEX software. The procedure includes self-calibration (flat-fielding), overlap correction, outlier rejection, and weighted coaddition into the final mosaic. Aperture photometry of all sources brighter than the 3- $\sigma$ noise of the local background was extracted using SExtractor. We also verified that the corresponding photometry agrees with the MIPS photometry from Fang et al. (2009) within the uncertainties.

\section{Results}

We are interested in the foreground population of the Orion A cloud, in particular the foreground populations toward the ONC. To separate it from the background we used the optical properties of dust grains in the Orion A cloud to block the optical light to the cloud background. This is a very effective way of isolating 

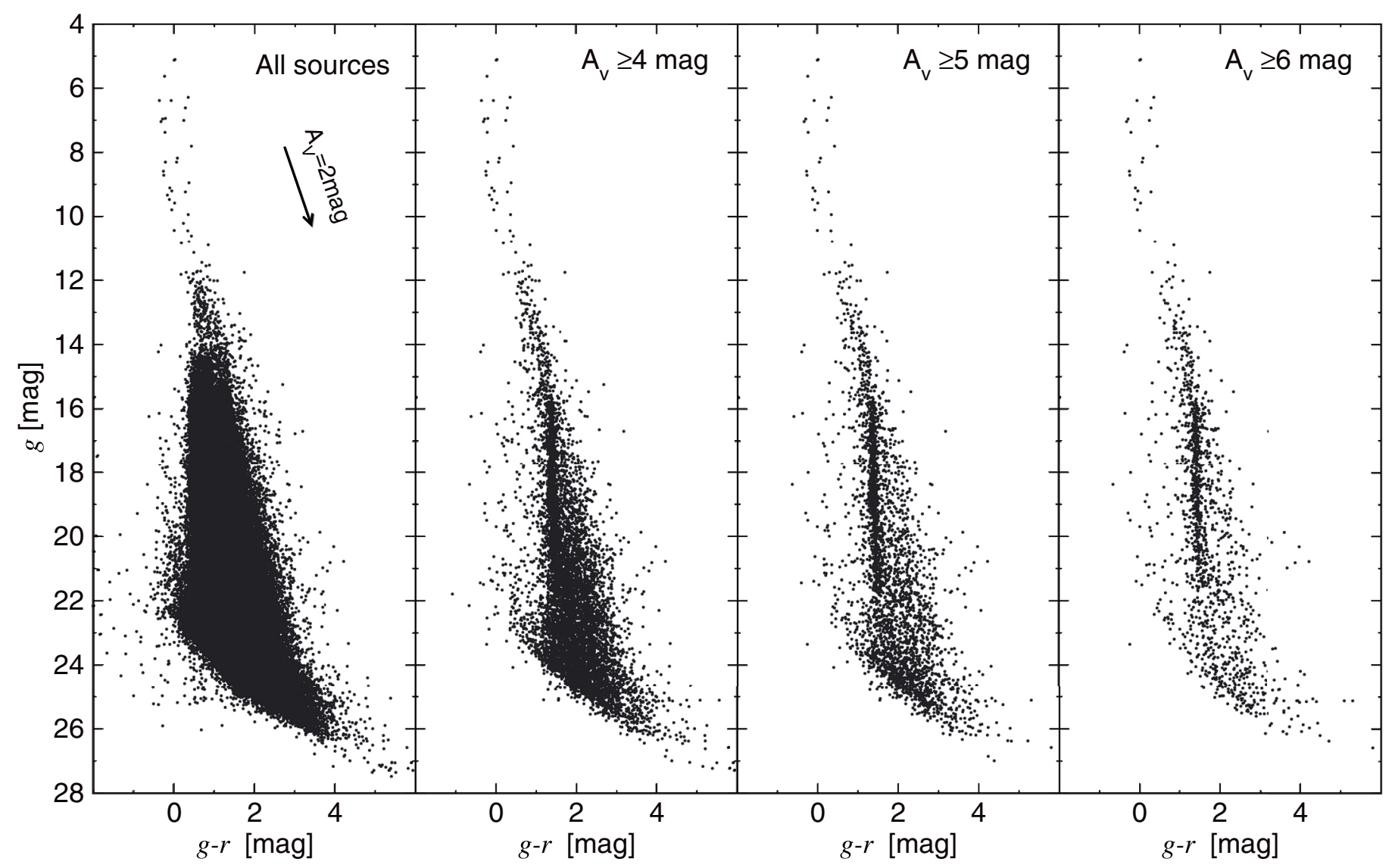

Fig. 3. $g$ vs. $g-r$ color-magnitude diagram for the entire survey in regions of increasing total line-of-sight extinction.

the stellar population between Earth and the Orion A cloud, in particular when using the blue optical bands where dust extinction is most effective. To select the final sample of foreground stars we made two filtering steps guided by color-magnitude and color-color diagrams in the optical and infrared. In particular, we 1) used blue optical magnitudes and colors to define a reliable subsample of sources in front of the cloud; then 2) employed a near-infrared color-color diagram to reject sources affected by extinction (these are sources that are either young stars inside the cloud or background sources that are bright enough to be detected in the optical survey).

The first filtering step is displayed in Fig. 3 where we present a $g$ vs. $g-r$ color-magnitude diagram combining the SDSS, CFHT/Megacam, and CAHA123 photometry. We chose $g-r$ over the more extinction-sensitive $u-g$ because the $u$-band observations are significantly shallower, and extremely sensitive to excesses related to accretion and activity.

The first diagram (on the left) represents the $g$ vs. $g-$ $r$ color-magnitude diagram for all sources in our combined database. The three color-magnitude diagrams to the right are a subset of the first, containing only sources projected against increasing contours of dust extinction of the Orion A cloud (about 4, 5, and 6 mag of visual extinction). These column density thresholds were estimated from the ${ }^{13} \mathrm{CO}$ map of Bally et al. (1987), cross-calibrated with the extinction map of Lombardi et al. (2011). While directly using the extinction map of Lombardi et al. (2011) gives similar results, we preferred to avoid dealing with any possible systematics affecting this map caused by a potential substantial population of foreground sources. Because we imposed the condition of keeping only sources that are seen against increasing levels of dust extinction, the number of stars naturally decreases because the solid angle on the sky decreases, and a clearly defined sequence appears. This sequence is not what is expected from the general Galactic population between Earth and the Orion A cloud at $400 \mathrm{pc}$, as confirmed with the Besaçon stellar population model (Robin et al. 2003). From this step we retained the subsample of sources that is seen in projection against column densities higher than $\sim 5$ visual magnitudes of extinction (third panel in Fig. 3), or a total of 2169 sources from more than $1.25 \times 10^{5}$ sources in the combined SDSS-Megacam-CAHA123 catalog. Most of the discarded sources have colors consistent with unreddened and slightly reddened unrelated field stars toward the background of the Orion A cloud. Among the sources that pass the first filter there could be some with $g$-band excess emission, but these should have a negligible effect in the selection process, in particular because the next filtering step is performed in the near-infrared.

The second filtering step consists of discarding extincted sources. We aimed to remove any source from the sample that might be associated with the cloud (YSOs still embedded in the cloud, for example), as well as background sources that are bright enough to be detected at $\sim 0.487 \mu \mathrm{m}$ ( $g$-band) through $A_{\mathrm{V}} \sim 5 \mathrm{mag}$ of cloud material. We performed this filtering with a $J-H$ vs. $H-K$ color-color diagram where extincted sources are easily identified along the reddening band, away from their unreddened main sequence (and giant) colors (e.g. Lada \& Adams 1992; Alves et al. 1998; Lombardi \& Alves 2001). We present in Fig. 4 the $J-H$ vs. $H-K$ color-color diagram for the 2169 sources that passed the first filtering step. The 


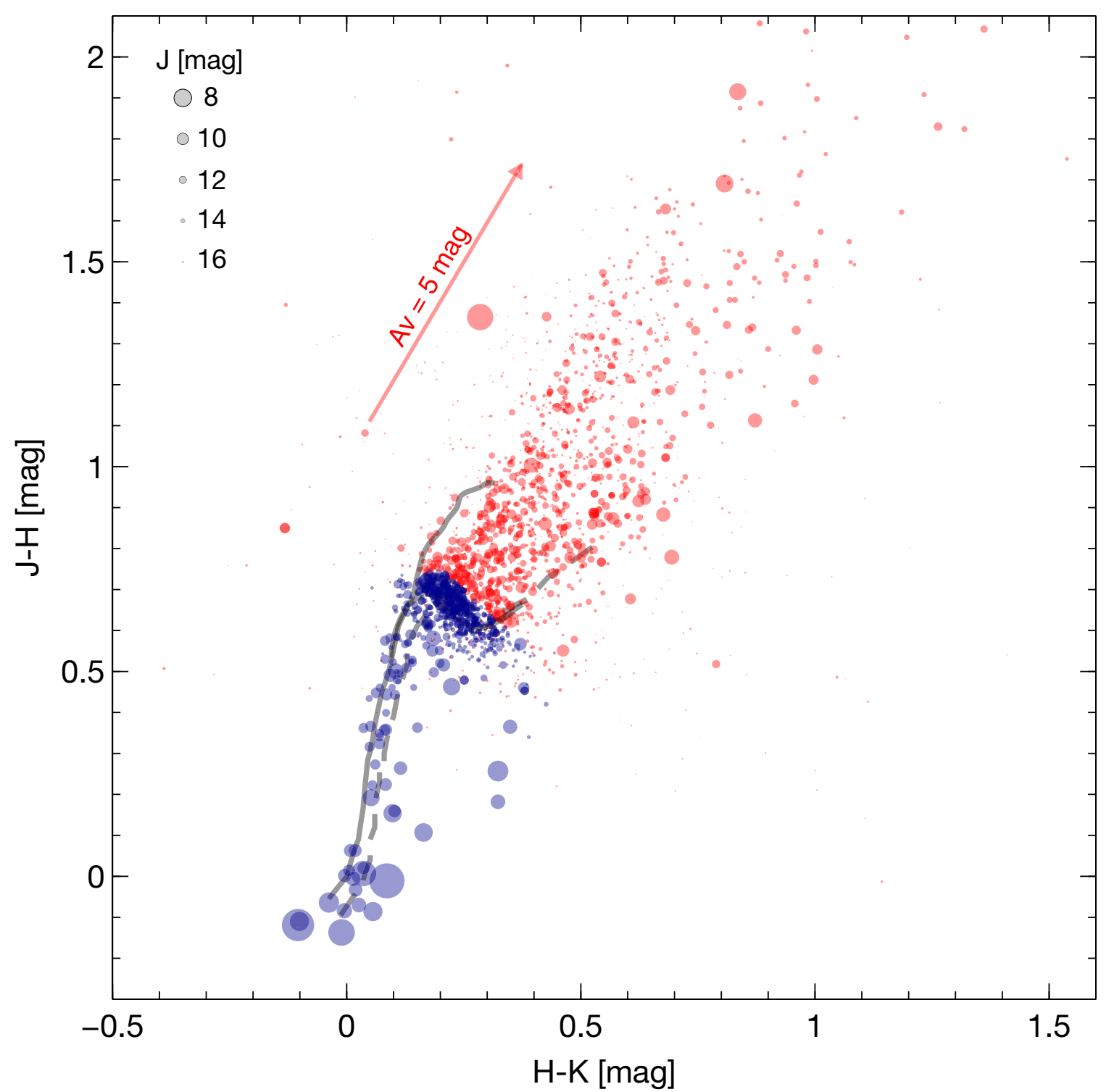

Fig. 4. $J-H$ versus $H-K$ color-color versus $J$ brightness diagram. The solid gray lines represent the main sequence and giant sequences from Bessell \& Brett (1988). The dashed gray line represents the main sequence from Kraus \& Hillenbrand (2007). The sizes of the symbols are proportional to the $J$-band brightness. Sources taken as foreground candidates are marked in blue, while rejected sources, mostly extincted sources, are marked in red.

size of the symbols in this figure is proportional to the $J$-band brightness. The selection criteria used to identify the likely foreground population were:

$H<\frac{0.96-(J-H)}{1.05} \operatorname{mag}$

$J<15 \mathrm{mag}$

$J-H<0.74 \cup H-K>-0.2 \cup H-K<0.43$ mag.

Sources that are consistent with having no extinction within the photometric errors are marked in blue, while rejected sources are marked in red. Condition (1), the main filter, is taken as the border between extincted and non-extincted sources, which was selected to be roughly parallel to the main-sequence early M-star branch (to about the color of a M4-M5 star). Condition (2) and (3) additionally reject sources that are faint or have dubious near-infrared colors (either bluer than physically possible, or redder than main-sequence stars, suggestive of a near-infrared excess). Condition (2) in particular makes the selection more robust against photometric errors (the typical photometric error imposed by condition (2) is $J_{\mathrm{err}} \sim 0.03 \pm 0.01$, $H_{\text {err }} \sim 0.03 \pm 0.01$, and $K_{\text {err }} \sim 0.03 \pm 0.01 \mathrm{mag}$, which translates into a maximum $A_{\mathrm{V}}$ error of $\sim 1 \mathrm{mag}$ ), and should reach a sensitivity limit capable of including M4 main-sequence stars at the distance of the cloud $(J \sim 15 \mathrm{mag})$. More than two thirds of the 2169 sources are rejected (red sources) and a total of 624 sources have colors consistent with foreground stars suffering no or negligible amounts of extinction ${ }^{1}$.

\subsection{Spatial distribution of the foreground and reddened sources}

Adding the third dimension ( $J$-band brightness) to Fig. 4 clearly brings into focus a well-populated main-sequence branch, from early types (B-stars) to late types (M-stars). The presence of so many early-type stars, as well as a clearly defined main

${ }_{1}$ A table of candidate foreground sources will be provided in Paper II. 
Foreground sources

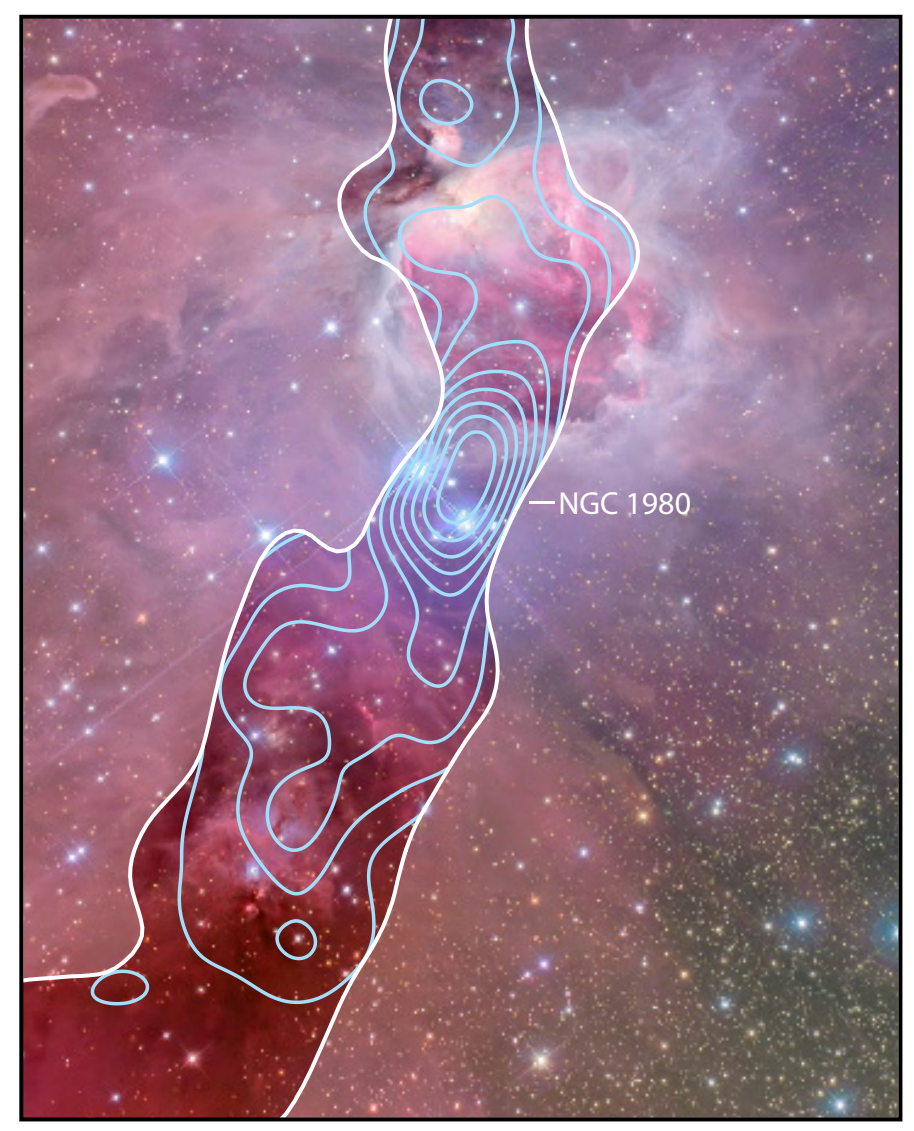

Reddened sources

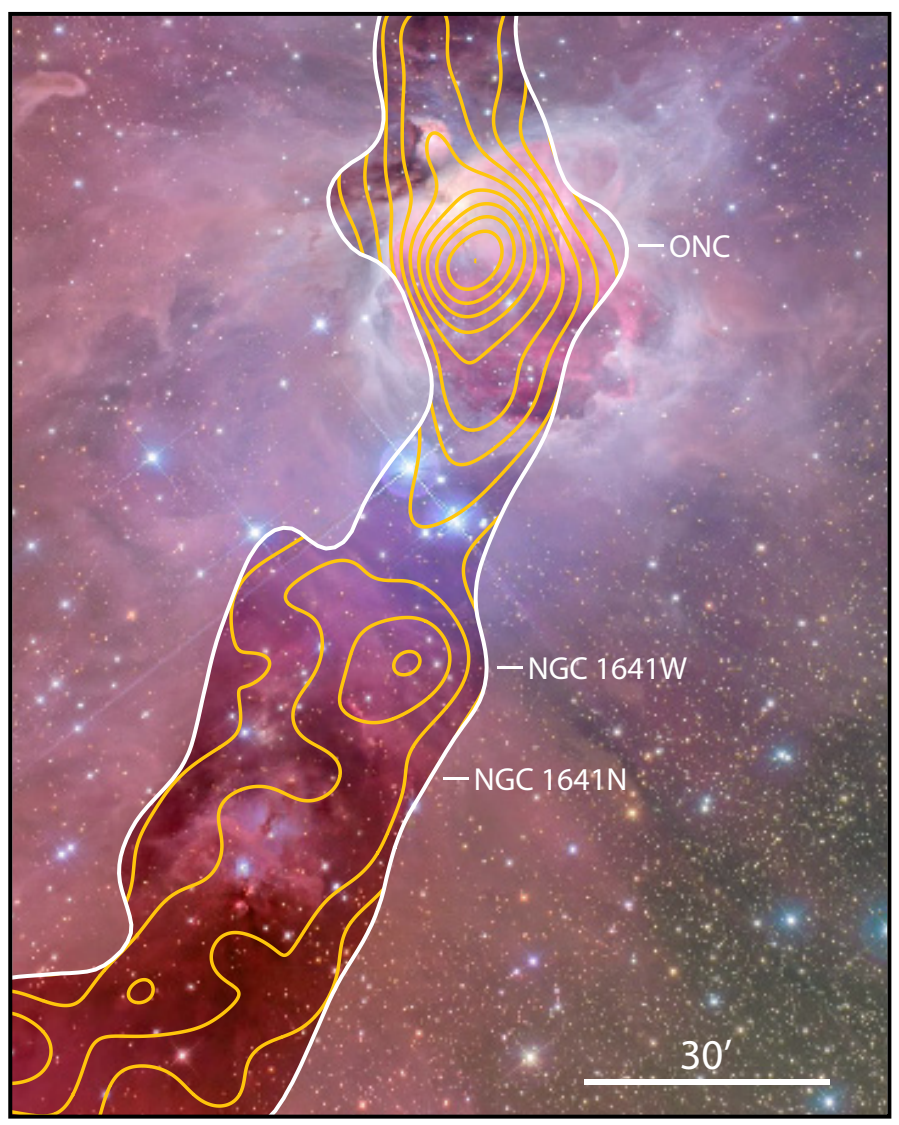

Fig. 5. Left panel: spatial density of the foreground sources (blue sample). The unshaded area in the figure represents the region of the cloud where $A_{\mathrm{V}} \geq 5 \mathrm{mag}$, on which the selection was performed. The blue contours (with increments of $10 \%$ from the maximum) represent the surface density of foreground sources (constructed with a Gaussian kernel with a width of $20^{\prime}$ ). Right panel: same as in the left panel but for the reddened sources. The distribution of foreground sources shows a clearly defined peak coinciding with the poorly studied NGC 1980 . The reddened sources, on the other hand, peak around 1) the Trapezium cluster and are mostly confined to the nebula and 2) the L1641N star-forming region, with a peak toward a hitherto unrecognized group of YSOs (see text). The reddened and foreground populations are spatially uncorrelated but there is significant overlap between the two, in particular with the sources inside the Orion nebula.

sequence, suggests that the foreground population is not dominated by the random Galactic field from Earth to the ONC region, but could instead be a well defined stellar population. To investigate this idea we present in the left panel of Fig. 5 the spatial density of foreground sources (blue sample). The unshaded area in the figure represents the region of the cloud where $A_{\mathrm{V}} \geq 5 \mathrm{mag}$, on which the selection was performed. We constructed a surface density map of foreground sources using a Gaussian kernel with a width of $20^{\prime}$ represented in the figure as blue contours (with increments of $10 \%$ from the maximum). It is clear from Fig. 5 that the distribution of foreground sources is not uniform, as expected for the Galactic field between Earth and the ONC region in the Orion A cloud, but is instead strongly peaked and fairly symmetric. The peak of the distribution coincides spatially with the poorly studied iota Ori cluster, or NGC 1980, suggesting that the foreground population is dominated by NGC 1980 cluster members. The elongated shape of the peak is not meaningful as it is caused by the relative narrow $A_{\mathrm{V}} \geq 5$ mag region on which the density was calculated.

The right panel of Fig. 5 shows the spatial distribution of reddened sources. This distribution is dominated by two peaks, a relatively well defined one in the Trapezium cluster region and another, more diffuse, coinciding with the overall gas distribution around the $\mathrm{L} 1641 \mathrm{~N}$ star forming region. This is not surprising as the reddened sources are expected to be dominated by the embedded YSOs in the Orion A cloud, and these are know to cluster around these two regions. More striking, instead, is that the foreground sources and the reddened sources appear spatially anti-correlated: the maximum of the distribution of the foreground sources coincides with the minimum of the distribution of reddened sources. This is evidence that the foreground population is not the emerging young stellar population from the ONC but is instead an entirely different population. Because it contains a fully sampled and unreddened main-sequence from B- to M-stars (see Fig. 4), this population is most likely the stellar population of the NGC 1980 cluster, seen in projection against the Orion A cloud. But because this population overlaps significantly with the ONC (the distribution of foreground sources appears symmetric to about $7 \mathrm{pc}$ from its center), it implies that the ONC is not comprised of a single cluster with the Trapezium as its core, but has instead three stellar populations: a) the youngest population, including the Trapezium and ongoing star formation associated with the dense gas in the nebula; b) part of the NGC 1980 cluster in its foreground; and c) the unrelated Galactic foreground and background population.

In Fig. 6 we present the distribution of all X-ray sources in the Third XMM-Newton serendipitous source catalog for the same region as shown in Fig. 5. We aim to investigate if 


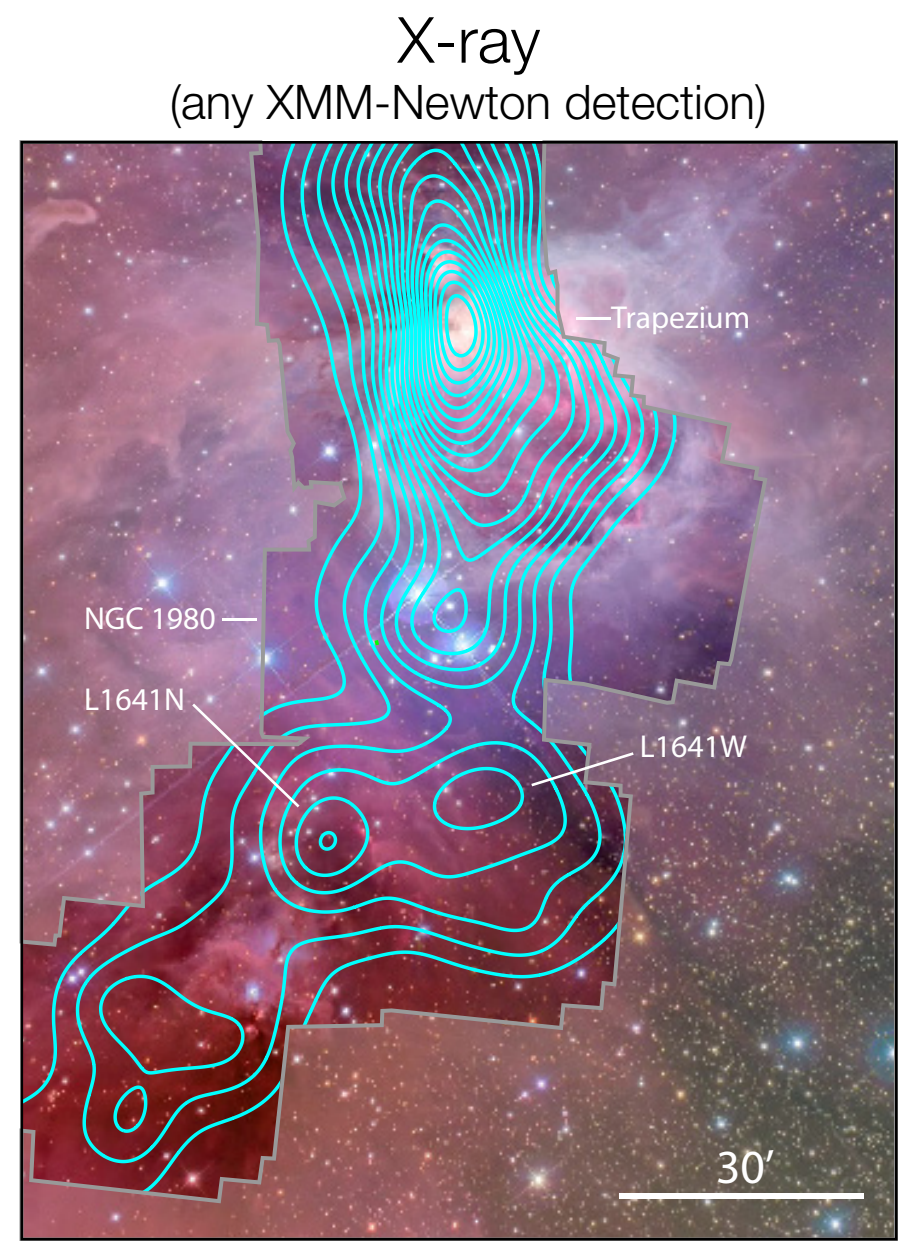

Fig. 6. Spatial density of all X-ray sources in the Third XMM-Newton serendipitous source catalog for the same region as in the previous figure. The Gaussian kernel used and contour separation are the same. NGC 1980 is detected as a distinct enhancement in the surface density of X-ray sources, together with the Trapezium cluster, the L1641N population, and the hitherto unrecognized group that we name L1641W.

the NGC 1980 X-ray source counts appear as a distinct peak in the surface density map (constructed using a Gaussian kernel with a width of $20^{\prime}$ as in the previous figure). As can be seen in this figure, both the foreground and the extincted populations are detected, and a distinct enhancement in the surface density of $\mathrm{X}$-ray sources is seen toward the center of NGC 1980, supporting the idea that the foreground population is not the emerging young stellar population from the ONC, but is instead an entirely different population. The highest peak in this surface density map is centered on the Trapezium cluster. This was not the case for the extincted population seen in Fig. 5, which peaks slightly to the south of the Trapezium, although this mismatch may arise simply because our Megacam $g$-band observations are affected by the bright nebula and dust extinction in the Trapezium region, hence are substantially less sensitive to the embedded population of the Trapezium cluster.

\subsubsection{A hitherto unrecognized group of YSOs?}

The enhancement of the reddened sources in Fig. 5 toward RA: $5^{\mathrm{h}} 35^{\mathrm{m}}$, Dec: $-6^{\circ} 18^{\mathrm{m}}$, immediately south of NGC 1980 and toward the west of what is normally taken as the L1641N cluster (e.g. Allen \& Davis 2008) is tantalizing. Could this relatively
Table 3. Position of clusters in Fig. 6, including the newly identified L1641W.

\begin{tabular}{lcc}
\hline \hline Field & RA (J2000) & Dec (J2000) \\
\hline L1641W & $05: 34: 51.0$ & $-06: 17: 40$ \\
NGC 1980 & $05: 35: 11.0$ & $-05: 58: 00$ \\
Trapezium & $05: 35: 16.5$ & $-05: 23: 14$ \\
L1641N & $05: 35: 55.7$ & $-06: 23: 55$ \\
\hline
\end{tabular}

small enhancement be another hitherto unrecognized group of YSOs? The enhancement is clearly detected in X-rays (Fig. 6) and is tentatively detected in the optical (Fig. 5), which supports this possibility. We name this potentially new group of about 50 stars (counted on the reddened sample) as L1641W. The group is not associated with any obvious nebula nor does it include any obvious bright star. Because it appears to be less extincted than the L1641N population, and it is not obviously detected in the Spitzer survey (e.g. Allen \& Davis 2008), it is probably more evolved than the L1641N population. We speculate that this new group is either a foreground young group ramming into the Orion A cloud, or a slightly older sibling of NGC $1641 \mathrm{~N}$, leaving the cloud.

\subsection{Luminosity function of foreground and reddened sources}

In Fig. 7 a we present the $K$-band luminosity functions for both the foreground (blue) and reddened (red) samples (see Fig. 4). To enable a direct comparison, the reddened sample was also constrained with condition (2), namely, $J<15$ mag. Surprisingly, the extincted sample (red) is brighter than the foreground sample (blue), even if no derredening procedure was applied to the red sample. We confirmed that the differences between the two luminosity functions are significant, to a $95 \%$ confidence level, by analyzing their empirical cumulative distribution functions (see Fig. 7b). Note that had we dereddened the extincted sample, the difference between the luminosity functions would have been even higher. This suggests, like in the previous section, that the foreground and reddened population are intrinsically different. A likely explanation for the difference in the luminosity functions is that the reddened sample is dominated by very young stellar objects that are still embedded the cloud, which are intrinsically brighter than normal stars because of stellar evolution and $K$-band excess emission.

\subsection{Velocity dispersion profile}

Tobin et al. (2009) presented an extensive kinematic study of the ONC, covering about $2^{\circ}$ of Declination centered on the Orion A cloud, from NGC 1977 down to L1641N. This survey builds on previous work by Fúrész et al. (2008) and constitutes the most complete and highest precision kinematic survey of this region to date, offering a unique possibility to characterize kinematically the ONC foreground population identified in this paper. In Fig. 8 we present the North-South velocity dispersion profile of the ONC region, taken from Table 13 of Tobin et al. (2009). The filled circles represent the sources in this paper with reliable radial velocities. The NGC 1977, the OMC2/3, the Trapezium cluster, and NGC 1980 are indicated, as well as the extent of the Orion nebula (light open circle). The thick red line represents the north-south velocity dispersion profile measured in bins of Declination (indicated by the thin horizontal lines). 

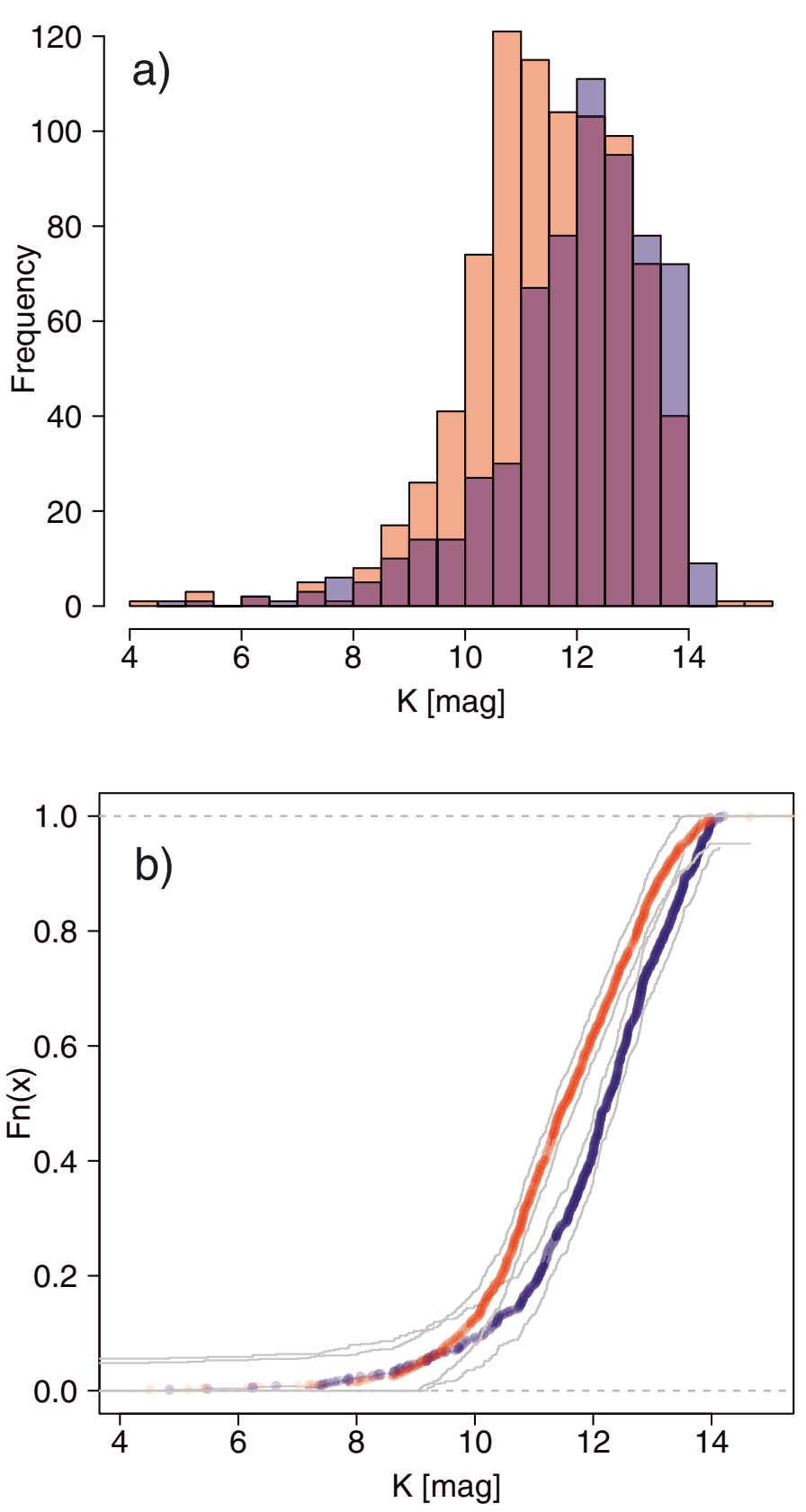

Fig. 7. a) $K$-band luminosity functions for the foreground (blue) and the extincted (red) sample (see Fig. 4). Although it is affected by extinction, the red sample is surprisingly brighter than the foreground sample (blue), suggesting that the two populations are intrinsically different. b) Empirical cumulative distribution functions for both samples, together with upper and lower simultaneous $95 \%$ confidence curves, confirming that the two populations have statistically different luminosity functions.

It is striking that the velocity dispersion profile has a minimum at the location of NGC 1980. This is perhaps the strongest indication we have that the stellar population of NGC 1980 is a distinct population from the reddened population inside Orion A. The measurement of the velocity dispersion in the bin that mostly includes NGC $1980\left(F W H M=2.1 \mathrm{~km} \mathrm{~s}^{-1}\right)$ was not optimized to isolate the most probable members of this cluster and should then be seen as an upper limit to the true velocity dispersion in this cluster. Still, this value is close to the velocity dispersion of the Trapezium cluster as measured from the

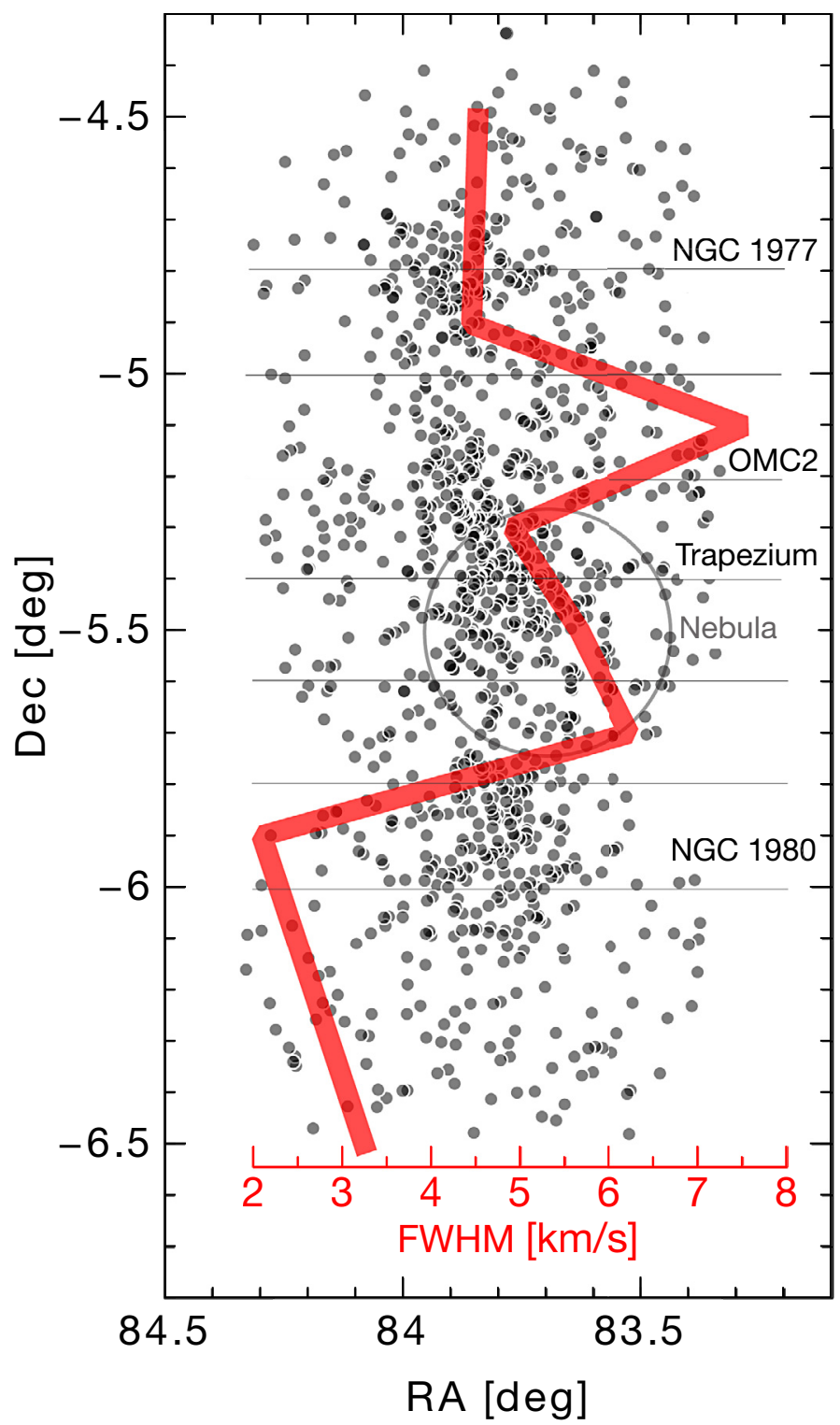

Fig. 8. North-south velocity dispersion profile of the ONC region. The filled circles represent the sources in Tobin et al. (2009) with reliable radial velocities. The NGC 1977, the Trapezium cluster, and NGC 1980 are indicated, as well as the extent of the Orion nebula (light open circle). The thick red line represents the north-south velocity dispersion profile measured in bins of Declination (indicated by the thin horizontal lines). There is an increase from the Trapezium to the edge of the nebula to the south, followed by a clear minimum around NGC 1980, strongly suggesting that NGC 1980 is a different population from the stellar population inside the nebula. Data from Table 13 of Tobin et al. (2009).

proper motion of stars within one half degree of the center of the Trapezium, namely, $1.34 \pm 0.18 \mathrm{~km} \mathrm{~s}^{-1}$ for a sample of brighter stars (van Altena et al. 1988) and $1.99 \pm 0.08 \mathrm{~km} \mathrm{~s}^{-1}$ for a larger sample including slightly fainter stars (Jones \& Walker 1988). Both velocity dispersions were corrected to the more recent estimate of the distance to the Orion A cloud (400 pc).

Given the striking differences in the velocity dispersion profile, we then calculated the mean radial velocity per bin from the subsample of single sources (not directly available in the Tobin et al. 2009, paper) and found that although showing variations from bin to bin, these variations are of the order of the 
measured dispersions. In particular, the mean velocity for the bin including the Trapezium $\left(-5.3^{\circ}<\delta<-5.4^{\circ}\right)$ and NGC 1980 $\left(-5.8^{\circ}<\delta<-6.0^{\circ}\right)$ is $25.7 \pm 3.0 \mathrm{~km} \mathrm{~s}^{-1}$ and $24.3 \pm 2.7 \mathrm{~km} \mathrm{~s}^{-1}$, respectively. Within the errors, estimated as the median absolute deviation in each bin, the NGC 1980 cluster has virtually the same radial velocity as the ONC. We note, however, that we were taking the bins as simple slices at constant declination, without trying to optimize their boundaries to better separate the different populations.

Because of the importance of measuring the velocity differences between the Trapezium and NGC 1980, especially for discussing the origin of NGC 1980, we made an alternative source selection and created two new subsamples that are in principle more pure, but have about three times fewer sources. For the NGC 1980 subsample we matched the Tobin et al. (2009) cata$\log$ with the foreground population identified in this paper. For the Trapezium we matched the Tobin et al. (2009) catalog with the COUP sample (Feigelson et al. 2002), which is dominated by Trapezium sources, and removed sources that matched the foreground population. Because this Trapezium subsample was meant to be of "high confidence", we used the radial velocity limits found in this subsample $\left(6.2 \mathrm{~km} \mathrm{~s}^{-1}\right.$ and $\left.36.6 \mathrm{~km} \mathrm{~s}^{-1}\right)$ to exclude five extreme outliers in the NGC 1980 sample (with velocities of $\sim-40$ and $\sim 90 \mathrm{~km} \mathrm{~s}^{-1}$ ). In these subsamples, the mean velocity for the Trapezium and NGC 1980 clusters is $25.4 \pm 3.0 \mathrm{~km} \mathrm{~s}^{-1}$ and $24.4 \pm 1.5 \mathrm{~km} \mathrm{~s}^{-1}$, respectively, or essentially the same values as derived above, with the important difference that the dispersion of velocities in NGC 1980 is now reduced by about a factor of two, once again suggesting that this cluster is a population distinct from the reddened population inside Orion, as argued above. Still, the measured velocity difference of $1 \mathrm{~km} \mathrm{~s}^{-1}$ is not statistically significant even with the decreased velocity dispersion.

\subsection{Age and population size of NGC 1980}

To estimate an age to the NGC 1980 cluster we compared the evolutionary status of class II sources in various clusters analyzing the median spectral energy distribution (SED) of latetype (spectral type later than K0) members. We followed the Hartmann et al. (2005) definition of Class II, namely objects with $0.2<[3.6]-[4.5]<0.7 \mathrm{mag}$ and $0.6<[5.8]-[8.0]<$ $1.1 \mathrm{mag}$. To compute the median SED for the different clusters we retrieved the optical, near-infrared (2MASS) and midinfrared (Spitzer and WISE) photometry for samples of confirmed members of Taurus (1-3 Myr, Luhman et al. 2010), IC 348 (1-3 Myr, Lada et al. 2006), NGC 1333 (1 Myr, Winston et al. 2010; Gutermuth et al. 2008), $\lambda$-Ori (5-7 Myr, Dolan \& Mathieu 2001; Barrado y Navascués et al. 2004), and $\eta$-Cha (5-10 Myr, Megeath et al. 2005). To compute the median SED for the Trapezium cluster we first defined a "high confidence" Trapezium member catalog, as we did in the previous section, by cross-matching the X-ray COUP sample from Prisinzano et al. (2008) with the foreground (NGC 1980) sample, and excluding all matches as unrelated foregrounds. The individual SEDs within each cluster were normalized to the $J$-band flux, and the median cluster SED of each cluster was computed. Figure 9 shows the result. One can see from this Fig. 9 that the optical part of the SED varies from cluster to cluster, mostly because of dust extinction. More striking, the mid-infrared ( $>3 \mu \mathrm{m})$ excesses, related to the presence of a disk, decrease systematically with age.

The median SED of NGC 1980 seems to fit between the median SED of Taurus (1-3 Myr) and $\lambda$-Ori (5-7 Myr), suggesting

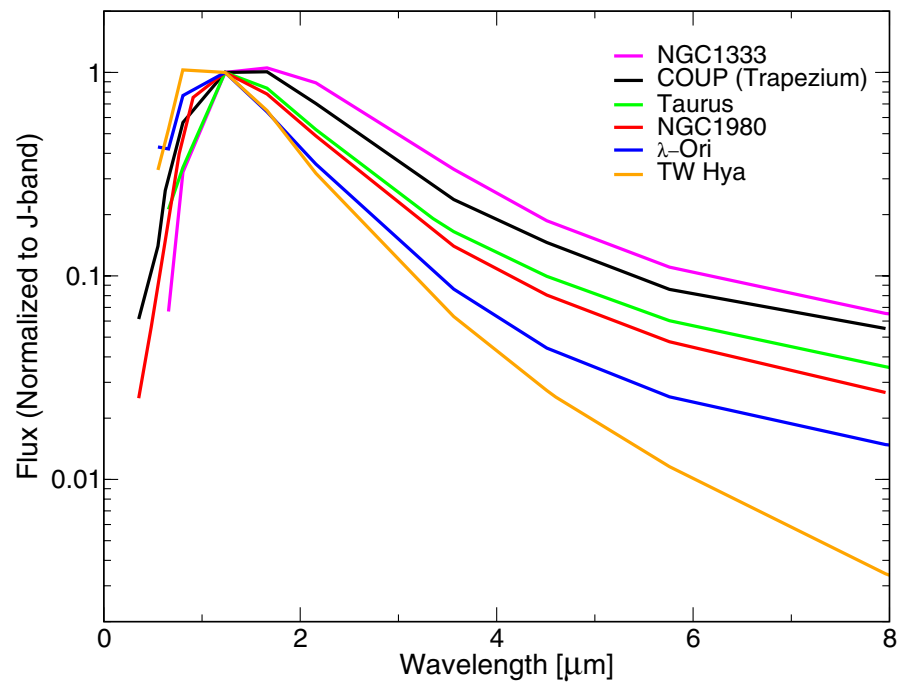

Fig. 9. Median SED of class II sources in clusters of various evolutionary stages: NGC 1333 (magenta, $1 \mathrm{Myr}$ ), Trapezium COUP sources (black, 1 Myr), Taurus (green, 1-3 Myr), NGC 1980 members (red), $\lambda$ Ori (blue 5-7 Myr) and $\eta$-Cha (orange, 5-10 Myr).

an age in between that of these regions. But another constraint is given by the massive stars in the center of the cluster. Of the five brightest stars at the peak of the spatial distribution in Fig. 5, only the brightest, iota Ori (O9 III, $V=2.77 \mathrm{mag}$ ), seems to have evolved from the main sequence. This implies an age of about 4-5 Myr for this star, assuming it started its life as a $25 M_{\odot}$ star (e.g. Massey 2003). This age fits well within the inferred age from the median SED and also agrees with the estimate of Warren \& Hesser (1978) for the age of Ori OB 1c subgroup (of about 4 Myr).

To estimate the size of the cluster population we focused on the distribution of foreground sources from the center of the cluster to the south in order to avoid incompleteness problems caused by the bright Orion nebula. We counted the number of sources falling on a $20^{\circ}$ "pie-slice" inside the $A_{\mathrm{V}} \geq 5$ mag region, centered on the cluster and with a radius of $7 \mathrm{pc}$. This radius approximately corresponds to the extent of the $10 \%$ contour in Fig. 5, chosen to account for contamination from the Galactic field between Earth and Orion (estimated to be $6-9 \%$ of the foreground population in Sect. 3.5.1). Note that this radius is not the half-mass radius but simply the radius to which we can trace the enhancement of sources over the unrelated foreground field. We repeated this measurement several times to account for uncertainties in the location of the cluster center and counted about 100 to 110 sources in the $20^{\circ}$ "pie-slice". Assuming spherical symmetry for the distribution of sources in NGC 1980, we expect a total of about 1800-2000 sources in NGC 1980, or a total cluster mass of about $1000 M_{\odot}$ (assuming an average mass per star of $0.5 M_{\odot}$ ).

Assuming NGC 1980 has a normal initial mass function (IMF), we can make a consistency test on the likelihood of the number of sources in this cluster being of the order of 2000. For this we constructed 200000 synthetic clusters of $1000 M_{\odot}$ each by randomly sampling the Kroupa and Chabrier IMFs (Kroupa 2001; Chabrier 2003) and tracked the mass of the most massive star in each synthetic cluster. The mean mass of the most massive star was $54 \pm 26 M_{\odot}$ (Kroupa) and $22 \pm 11 M_{\odot}$ (Chabrier). Assuming there were no supernovae in NGC 1980 yet, and that iota Ori is the most massive star in the cluster, then, to first 
approximation, a population of about 2000 sources seems a plausible estimate of the size of the NGC 1980 population.

At the moment we cannot derive a reliable cluster radial profile nor a half-mass radius, or even be certain about the position of the center of the cluster, because our optical observations are incomplete in the vicinity of the early-type stars of NGC 1980, and we only have a "pie-slice" view of the radial extent of the cluster. This should be improved in follow-up work, in particular in combination with dedicated near-infrared observations, which are less sensitive to the strong brightness contrasts between early- and late-type stars in this cluster.

\subsubsection{Origin of NGC 1980 and its impact on the Orion A cloud}

We found in Sect. 3.3 that the radial velocity of NGC 1980 is indistinguishable, or has a difference of the order of a few $\mathrm{km} \mathrm{s}^{-1}$ at best, from the radial velocity of the embedded Trapezium population. This surprising result implies that the radial velocity of NGC 1980 is essentially the same as the velocity of the gas in the Orion A cloud, since the ONC population has the same radial velocity as the cloud (Tobin et al. 2009). This strongly suggests that NGC 1980 is somehow connected to the Orion A cloud, or better, that the cloud that formed NGC 1980 was physically related to the current Orion A cloud. One would not expect the distance to NGC 1980 to be substantially different than the current distance estimate to the ONC, and a fitting of the ZAMS on the optical data presented in this paper is indeed consistent with a distance of $400 \mathrm{pc}$.

Despite its relatively older age, lack of obvious $\mathrm{H}_{\text {II }}$ region, and lack of measurable dust extinction, NGC 1980 moves away from Earth at the same velocity as the large Orion A cloud on which it is seen in projection. Because of their likely proximity, one wonders about the effects of the ionizing stars from NGC 1980 on the Orion A cloud, or what the cloud was about 4-5 Myr ago, in particular about a possible acceleration and compression of the cloud by the UV radiation from these stars. How significant was/is this process in this region? Could the formation of the ONC have been triggered by its older sibling, as suggested in Bally (2008)? At first glance our results seem to argue that the impact would have been minimal, NGC 1980 has essentially the same radial velocity as the Orion A cloud, but the work of Oort (1954), Kahn (1954), and Oort \& Spitzer (1955) suggests that final speeds between the ionizing star and the cloud would be of the order of a few $\mathrm{km} \mathrm{s}^{-1}$, which cannot be ruled out by the current accuracy of the data. While our results do not give final evidence in support of the tantalizing suggestion that the formation of the ONC could have been triggered by NGC 1980, they are not inconsistent with it either. A new dedicated radial velocity survey of the region, together with a sensitive proper motion survey, are needed to understand the interplay between these two massive clusters. This configuration (an embedded cluster in the vicinity of an $\sim 5$ Myr cluster) is unlikely to be unique in massive star-forming clouds, but it will best addressed in the nearest example.

\subsection{Contamination of ONC catalogs}

We have shown above that there is a rich and distinct foreground population of stars, likely associated with the young ( $5 \mathrm{Myr}$ ) poorly studied but massive NGC 1980 cluster, which is not directly associated with the ongoing star formation in the ONC. This finding raises concerns on the contamination of currently available observables for this important region, and future studies should take this foreground population into account. But how strong is this contamination? There are two well-known ONC catalogs used in the literature, namely Hillenbrand (1997) and the catalogs of Da Rio et al. (2009), Da Rio et al. (2010), and Da Rio et al. (2012) which cover a roughly square area of about $0.5^{\circ} \times 0.5^{\circ}(\sim 3.5 \times 3.5 \mathrm{pc})$ centered on the Trapezium cluster. The Da Rio et al. (2012) catalog supersedes all previous cata$\log$, but it is the most recent, hence least used in the community. On the other hand, the Hillenbrand (1997) catalog has been used extensively in the literature and has spawned a large number of the star formation studies on the star formation properties of the $\mathrm{ONC}$ region. We estimate here the likely foreground contamination fraction for the Hillenbrand (1997) catalog because it is the most used one, but also because it is likely to be the least contaminated since the Da Rio catalogs cover a slightly larger area of the sky toward NGC 1980.

To estimate the probable contamination fraction of Hillenbrand (1997), we matched the foreground population with this catalog for stars falling within the $A_{\mathrm{V}} \geq 5$ mag region where the foreground was selected (see Fig. 5) and where $I$-band $<16$ mag. The last constraint accounts for the fact that the Hillenbrand (1997) sample is not uniformly deep (it reaches about 2 mag deeper around the Trapezium cluster), and that the selection of foreground stars, made at $g$-band, seems complete to about $I$-band $\sim 16$ mag (after transformation of the SDSS photometry into Johnson's, Ivezić et al. 2007). We find that $11 \%$ of the sources in the Hillenbrand (1997) catalog have a match in the foreground sample ( $8 \%$ if we remove the constraint on the $I$-band brightness). If one sees the Trapezium cluster as a component of the ONC, and not as the only component, and excludes it from consideration, the fraction of foreground contaminants in the $\mathrm{ONC}$ rises to $32 \%$. For this estimate the area on the sky covered by the Trapezium cluster is taken as an ellipse with $a=7.5^{\prime}$ and $b=3.8^{\prime}$, with a position angle of $-10^{\circ}$, similar to the definition in Hillenbrand \& Hartmann (1998). One can also estimate the possible contamination to the entire Hillenbrand (1997) catalog by applying Eq. (1) to it, which yields a contamination fraction of $20 \%$, or $63 \%$ when the Trapezium is excluded from consideration. Note that all these estimates assume that the fraction of ONC stars without measurable extinction is negligible, which is likely given the distribution of foreground stars in Fig. 5, but will need to be investigated further in future work.

Even including the Trapezium cluster in the consideration, contamination fractions of about $10-20 \%$ are significant and will necessarily lead to systematic errors in the basic derived physical quantities for this star formation benchmark. Still, these are necessarily lower limits to the true contamination fraction of the ONC sample for at least two reasons: 1) our $g$-band Megacam survey is not as sensitive in regions of high nebular brightness, especially around the Trapezium; and 2) we are not sensitive, by design, to background sources. While it is normally argued that the high background extinction behind the Trapezium blocks most background stars, this is only valid for the inner regions of the Trapezium cluster $\left(\sim 25^{\prime 2}\right)$, but not valid for the entire $\sim 700^{\prime 2}$ Orion nebula, (e.g. Johnstone \& Bally 1999; Buckle et al. 2012). So background contamination is variable across the ONC and expected for any optical or infrared survey of this region. With regard to the contamination of the ONC region by NGC 1980, it is a function of the position in the nebula, which is at a minimum at the center of the Trapezium where the Trapezium cluster stellar density is highest, gradually increasing toward the south as one approaches the core of NGC 1980 (see Fig. 5). 

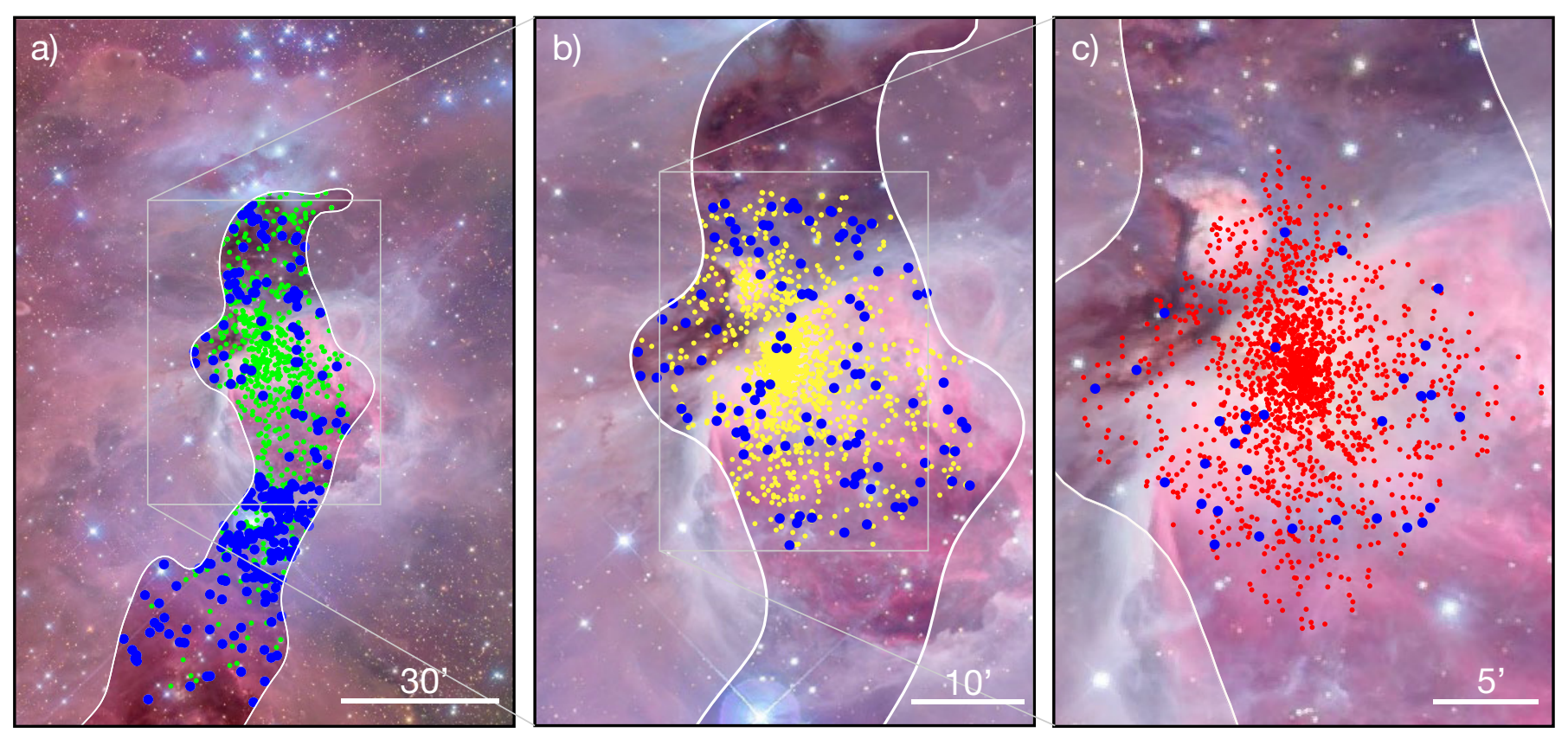

Fig. 10. Matches between the foreground contamination and well-known catalogs of the ONC. a) In green, the optical spectroscopic survey of Tobin et al. (2009), matches with the foreground population are marked in blue. b) In yellow, the well-studied sample of Hillenbrand (1997), matches with the foreground population are marked in blue. c) In red, the sample of X-ray sources from the COUP project (Feigelson et al. 2002), matches with the foreground population are marked in blue. As in Fig. 5, the unshaded area represents the region of the cloud where $A_{\mathrm{V}} \geq 5$ mag, on which the selection of foreground sources was performed.

\subsubsection{Unrelated Galactic field foreground population}

Most of the identified foreground population (624 sources) is likely to belong to NGC 1980 , as seen from the symmetric and peaked spatial distribution in Fig. 5, but some fraction of these must consist of the Galactic field population between Earth and the Orion A cloud. A first and simple estimate of the size of this population can be made by correlating the foreground population with the sample of Tobin et al. (2009) for which good radial velocity measurements exist (see Fig. 10). The distribution of radial velocities of the 188 sources in common to both populations reveals a Gaussian like distribution centered at $\sim 26.1 \mathrm{~km} \mathrm{~s}^{-1}$, with a gap roughly between $-20-0 \mathrm{~km} \mathrm{~s}^{-1}$ and $40-60 \mathrm{~km} \mathrm{~s}^{-1}$ without any source, and three sources below $-20 \mathrm{~km} \mathrm{~s}^{-1}$ and nine above $60 \mathrm{~km} \mathrm{~s}^{-1}$ (i.e., 12 potential outliers). Not surprisingly, if we sigma-clip the entire distribution at $3 \sigma$, we find 11 outliers. If we remove the 12 potential outliers from the distribution described above and then sigma-clip the rest of the distribution, we find five more outliers, but this time at the wings of the distribution. This suggests that about 6-9\% of the foreground sources identified in this work are likely field sources unrelated to NGC 1980. This estimate of the Galactic field foreground contamination for Orion A roughly agrees with what would be expected from the Besaçon stellar population model (Robin et al. 2003) for the depth of our Megacam survey.

\section{Conclusions}

We have made a link between the foreground population toward the well-known star formation benchmark ONC region and the stellar population of the poorly studied NGC 1980 cluster (or iota Ori cluster). Not only did we detect a well-populated main-sequence (from B-stars to M-stars), the foreground sources have 1) a defined spatial distribution that peaks near iota Ori; 2) a fainter luminosity function when compared to the extincted young population embedded inside the cloud; and 3) a lowvelocity dispersion, typical of that of other young clusters.

Unlike the ONC, NGC 1980 is a slightly older cluster (4-5 Myr), lacks an obvious $\mathrm{H}_{\mathrm{II}}$ region, and is comparatively free of dust extinction. Surprisingly, the radial velocity of NGC 1980 is currently indistinguishable from the radial velocity of the ONC embedded population or the radial velocity of Orion A cloud, suggesting that both clusters are genetically related. They are situated at about the same distance from Earth.

A general concern that this study raises is the risk of population mixing in star formation studies. It is unlikely that the ONC is atypical in this respect, and a dedicated multiwavelength study to separate the different populations, together with a sensitive proper motion survey of the region, is urgently needed. The ONC is still the closer massive star formation region to Earth, and albeit more complicated than first assumed, it is still the one offering the best detailed view on the formation of massive stars and clusters.

To summarize, we used the optical effects of dust extinction to block the background stellar population of the Orion A cloud, and found that there is a rich foreground stellar population in front of the cloud, in particular the ONC. This population contains a well populated main-sequence, from B-stars to M-stars.

The spatial distribution of the foreground population is not random but clusters strongly around NGC 1980 (iota Ori), has a fainter luminosity function, and a different velocity dispersion from the reddened population inside the Orion A cloud. This foreground population is, in all likelihood, the extended stellar content of the poorly studied NGC 1980 cluster.

We estimate the number of members of NGC 1980 to be about 1800-2000, which makes it one of the most massive 
clusters in the entire Orion complex, and estimate its age to be 4-5 Myr by comparing the median SEDs among known young populations and constraints from the age of the postmain-sequence star iota Ori.

This newly found population overlaps significantly with what is currently assumed to be the ONC and the L1641N populations, and can make up for more than 10-20\% of what is currently taken as the ONC population (30-60\% excluding the Trapezium cluster).

Our results suggest that what is normally considered as the ONC in the literature should be seen as a mix of several unrelated populations: 1) the youngest population, including the Trapezium cluster and ongoing star formation in the dense gas inside the nebula; 2) the young foreground population, dominated by the NGC 1980 cluster; and 3) the poorly constrained population of foreground and background Galactic field stars.

We re-determined the mean radial velocity for the Trapezium and NGC 1980 clusters to be $25.4 \pm 3.0 \mathrm{~km} \mathrm{~s}^{-1}$ and $24.4 \pm$ $1.5 \mathrm{~km} \mathrm{~s}^{-1}$, respectively, or indistinguishable within the errors, and similar to the radial velocity of the Orion A cloud, suggestive of a genetical connection between the two.

We identified a hitherto unrecognized group of about 50 YSOs west of L1641N (L1641W) that we speculate is either a foreground group ramming into the Orion A cloud, or a slightly older sibling of NGC $1641 \mathrm{~N}$, leaving the cloud.

This work supports a scenario where the ONC and L1641N are not directly associated with NGC 1980, i.e., they are not the same population emerging from its parental cloud but are instead distinct overlapping populations. This calls for a revision of most of the observables in the benchmark ONC region (e.g., ages, age spread, mass function, disk frequency, etc.).

Acknowledgements. We thank the referee, John Bally, for comments that improved the manuscript. We also thank John Tobin, Nicola Da Rio, and Lynne Hillenbrand for comments and clarifications that improved the presentation of results. H. Bouy is funded by the Ramón y Cajal fellowship program number RYC-2009-04497. We acknowledge support from the Faculty of the European Space Astronomy Centre (ESAC). This publication is supported by the Austrian Science Fund (FWF). We thank the Calar Alto Observatory for allocation of director's discretionary time to this program. Funding for SDSSIII has been provided by the Alfred P. Sloan Foundation, the Participating Institutions, the National Science Foundation, and the US Department of Energy Office of Science. The SDSS-III web site is http://www.sdss3. org/. SDSS-III is managed by the Astrophysical Research Consortium for the Participating Institutions of the SDSS-III Collaboration including the University of Arizona, the Brazilian Participation Group, Brookhaven National Laboratory, University of Cambridge, University of Florida, the French Participation Group, the German Participation Group, the Instituto de Astrofisica de Canarias, the Michigan State/Notre Dame/JINA Participation Group, Johns Hopkins University, Lawrence Berkeley National Laboratory, Max Planck Institute for Astrophysics, New Mexico State University, New York University, Ohio State University, Pennsylvania State University, University of Portsmouth, Princeton University, the Spanish Participation Group, University of Tokyo, University of Utah, Vanderbilt University, University of Virginia, University of Washington, and Yale University. This publication makes use of data products from the Widefield Infrared Survey Explorer, which is a joint project of the University of California, Los Angeles, and the Jet Propulsion Laboratory/California Institute of Technology, funded by the National Aeronautics and Space Administration. Based on observations obtained with XMM-Newton, an ESA science mission with instruments and contributions directly funded by ESA Member States and NASA. This research used the facilities of the Canadian Astronomy Data Centre operated by the National Research Council of Canada with the support of the Canadian Space Agency. This publication makes use of data products from the Two Micron All Sky Survey, which is a joint project of the University of Massachusetts and the Infrared Processing and Analysis Center/California Institute of Technology, funded by the National Aeronautics and Space Administration and the National Science Foundation. This work is based in part on observations made with the Spitzer Space Telescope, which is operated by the Jet Propulsion Laboratory, California Institute of Technology under a contract with NASA.

\section{References}

Allen, L., \& Davis, C. 2008, Low Mass Star Formation in the Lynds 1641 Molecular Cloud, ed. B. Reipurth, 621

Allison, R. J., \& Goodwin, S. P. 2011, MNRAS, 415, 1967

Alves, J., Lada, C. J., Lada, E. A., Kenyon, S. J., \& Phelps, R. 1998, ApJ, 506, 292

Bally, J. 2008, Overview of the Orion Complex, ed. B. Reipurth, 459

Bally, J., Lanber, W. D., Stark, A. A., \& Wilson, R. W. 1987, ApJ, 312, L45

Barrado y Navascués, D., Stauffer, J. R., Bouvier, J., Jayawardhana, R., \& Cuillandre, J.-C. 2004, ApJ, 610, 1064

Bate, M. R., Bonnell, I. A., \& Bromm, V. 2003, MNRAS, 339, 577

Bertin, E. 2010a, in JENAM 2010, Joint European and National Astronomy Meeting

Bertin, E. 2010b, Astrophysics Source Code Library, 10068

Bertin, E., \& Arnouts, S. 1996, A\&AS, 117, 393

Bessell, M. S., \& Brett, J. M. 1988, PASP, 100, 1134

Blaauw, A. 1964, ARA\&A, 2, 213

Bonnell, I. A., Bate, M. R., Clarke, C. J., \& Pringle, J. E. 2001, MNRAS, 323, 785

Boulade, O., Charlot, X., Abbon, P., et al. 2003, in SPIE Conf. Ser., 4841, eds. M. Iye, \& A. F. M. Moorwood, 72

Briceño, C., Preibisch, T., Sherry, W. H., et al. 2007, Protostars and Planets V, 345

Brown, A. G. A., de Geus, E. J., \& de Zeeuw, P. T. 1994, A\&A, 289, 101

Buckle, J. V., Davis, C. J., Francesco, J. D., et al. 2012, MNRAS, 422, 521

Chabrier, G. 2003, PASP, 115, 763

Clarke, C. J., Bonnell, I. A., \& Hillenbrand, L. A. 2000, Protostars and Planets IV, 151

Cuillandre, J.-C., Magnier, E. A., Isani, S., et al. 2004, in Scientific Detectors for Astronomy, The Beginning of a New Era, eds. P. Amico, J. W. Beletic, \& J. E. Belectic, Astrophys. Space Sci. Lib., 300, 287

Cutri, R. M., \& et al. 2012, VizieR Online Data Catalog, 2311, 0

Da Rio, N., Robberto, M., Soderblom, D. R., et al. 2009, ApJS, 183, 261

Da Rio, N., Robberto, M., Soderblom, D. R., et al. 2010, ApJ, 722, 1092

Da Rio, N., Robberto, M., Hillenbrand, L. A., Henning, T., \& Stassun, K. G. 2012, ApJ, 748, 14

Devillard, N. 1997, The Messenger, 87, 19

Dolan, C. J., \& Mathieu, R. D. 2001, AJ, 121, 2124

Fang, M., van Boekel, R., Wang, W., et al. 2009, A\&A, 504, 461

Feigelson, E. D., Broos, P., Gaffney, III, J. A., et al. 2002, ApJ, 574, 258

Feigelson, E. D., Gaffney, III, J. A., Garmire, G., Hillenbrand, L. A., \& Townsley, L. 2003, ApJ, 584, 911

Fưrész, G., Hartmann, L. W., Megeath, S. T., Szentgyorgyi, A. H., \& Hamden, E. T. 2008, ApJ, 676, 1109

Gomez, M., \& Lada, C. J. 1998, AJ, 115, 1524

Gutermuth, R. A., Myers, P. C., Megeath, S. T., et al. 2008, ApJ, 674, 336

Hartmann, L., Megeath, S. T., Allen, L., et al. 2005, ApJ, 629, 881

Herbig, G. H., \& Terndrup, D. M. 1986, ApJ, 307, 609

Herbst, W., Bailer-Jones, C. A. L., Mundt, R., Meisenheimer, K., \& Wackermann, R. 2002, A\&A, 396, 513

Hillenbrand, L. A. 1997, AJ, 113, 1733

Hillenbrand, L. A., \& Carpenter, J. M. 2000, ApJ, 540, 236

Hillenbrand, L. A., \& Hartmann, L. W. 1998, ApJ, 492, 540

Hillenbrand, L. A., Strom, S. E., Calvet, N., et al. 1998, AJ, 116, 1816

Hirota, T., Bushimata, T., Choi, Y. K., et al. 2007, PASJ, 59, 897

Hsu, W.-H., Hartmann, L., Allen, L., et al. 2012, ApJ, 752, 59

Huff, E. M., \& Stahler, S. W. 2006, ApJ, 644, 355

Ivezić, Ž., Smith, J. A., Miknaitis, G., et al. 2007, AJ, 134, 973

Jeffries, R. D., Littlefair, S. P., Naylor, T., \& Mayne, N. J. 2011, MNRAS, 418, 1948

Johnson, H. M. 1965, ApJ, 142, 964

Johnstone, D., \& Bally, J. 1999, ApJ, 510, L49

Jones, B. F., \& Walker, M. F. 1988, AJ, 95, 1755

Kahn, F. D. 1954, Bull. Astron. Inst. Netherlands, 12, 187

Kim, M. K., Hirota, T., Honma, M., et al. 2008, PASJ, 60, 991

Klessen, R. S., \& Burkert, A. 2000, ApJS, 128, 287

Köhler, R., Petr-Gotzens, M. G., McCaughrean, M. J., et al. 2006, A\&A, 458, 461

Kraus, A. L., \& Hillenbrand, L. A. 2007, AJ, 134, 2340

Kroupa, P. 2001, MNRAS, 322, 231

Krumholz, M. R., Klein, R. I., \& McKee, C. F. 2011, ApJ, 740, 74

Lada, C. J., \& Adams, F. C. 1992, ApJ, 393, 278

Lada, E. A., \& Lada, C. J. 1995, AJ, 109, 1682

Lada, C. J., Muench, A. A., Haisch, Jr., K. E., et al. 2000, AJ, 120, 3162

Lada, C. J., Muench, A. A., Luhman, K. L., et al. 2006, AJ, 131, 1574

Lada, C. J., Lombardi, M., \& Alves, J. F. 2010, ApJ, 724, 687 
Lombardi, M., \& Alves, J. 2001, A\&A, 377, 1023

Lombardi, M., Alves, J., \& Lada, C. 2011, A\&A, 535, A16

Luhman, K. L., Allen, P. R., Espaillat, C., Hartmann, L., \& Calvet, N. 2010, ApJS, 186, 111

Magnier, E. A., \& Cuillandre, J.-C. 2004, PASP, 116, 449

Makovoz, D., \& Marleau, F. R. 2005, PASP, 117, 1113

Massey, P. 2003, ARA\&A, 41, 15

Megeath, S. T., Hartmann, L., Luhman, K. L., \& Fazio, G. G. 2005, ApJ, 634, L113

Menten, K. M., Reid, M. J., Forbrich, J., \& Brunthaler, A. 2007, A\&A, 474, 515

Muench, A. A., Alves, J., Lada, C. J., \& Lada, E. A. 2001, ApJ, 558, L51

Muench, A. A., Lada, E. A., Lada, C. J., \& Alves, J. F. 2002, ApJ, 573, 366

Muench, A., Getman, K., Hillenbrand, L., \& Preibisch, T. 2008, Star Formation in the Orion Nebula I: Stellar Content, ed. B. Reipurth, 483

O'dell, C. R., Wen, Z., \& Hu, X. 1993, ApJ, 410, 696

O’Dell, C. R., Muench, A., Smith, N., \& Zapata, L. 2008, Star Formation in the Orion Nebula II: Gas, Dust, Proplyds and Outflows, ed. B. Reipurth, 544

Oort, J. H. 1954, Bull. Astr. Instit. Nether., 12, 177

Oort, J. H., \& Spitzer, L. J. 1955, ApJ, 121, 6
Palla, F., \& Stahler, S. W. 1999, ApJ, 525, 772

Petr, M. G., Coudé du Foresto, V., Beckwith, S. V. W., Richichi, A., \& McCaughrean, M. J. 1998, ApJ, 500, 825

Prisinzano, L., Micela, G., Flaccomio, E., et al. 2008, ApJ, 677, 401

Reggiani, M., Robberto, M., Da Rio, N., et al. 2011, A\&A, 534, A83

Robin, A. C., Reylé, C., Derrière, S., \& Picaud, S. 2003, A\&A, 409, 523

Sandstrom, K. M., Peek, J. E. G., Bower, G. C., Bolatto, A. D., \& Plambeck, R. L. 2007, ApJ, 667, 1161

Skrutskie, M. F., Cutri, R. M., Stiening, R., et al. 2006, AJ, 131, 1163

Smith, J. A., Tucker, D. L., Kent, S., et al. 2002, AJ, 123, 2121

Tan, J. C., Krumholz, M. R., \& McKee, C. F. 2006, ApJ, 641, L121

Tobin, J. J., Hartmann, L., Furesz, G., Mateo, M., \& Megeath, S. T. 2009, ApJ, 697,1103

van Altena, W. F., Lee, J. T., Lee, J.-F., Lu, P. K., \& Upgren, A. R. 1988, AJ, 95 1744

Vicente, S. M., \& Alves, J. 2005, A\&A, 441, 195

Walker, M. F. 1972, ApJ, 175, 89

Warren, Jr., W. H., \& Hesser, J. E. 1978, ApJS, 36, 497

Watson, M. G., Schröder, A. C., Fyfe, D., et al. 2009, A\&A, 493, 339

Winston, E., Megeath, S. T., Wolk, S. J., et al. 2010, AJ, 140, 266 